\title{
ASERTIVIDAD Y ANSIEDAD EN UNIVERSITARIOS MEXICANOS Y NORTEAMERICANOS. UN ESTUDIO PILOTO
}

\author{
Por \\ Ángel Manuel Ramírez Peredo* \\ Teresa Mange Cabrillas **
}

\begin{abstract}
RESUMEN
A través de una encuesta aplicada a tres grupos de estudiantes universitarios de ambos lados de la frontera México-Estados Unidos (mexicanos, chicanos y norteaméricanos), en este trabajo se trata de medir la asertividad, que es una respuesta ante situaciones que provocan ansiedad y consiste en la defensa de los derechos personales pero manteniendo una actitud de respecto ante el derecho de los demás.

Los resultados indican un elevado nivel de ansiedad y un notable decremento en asertividad en el grupo de los chicanos; además, para los tres grupos en el sexo femenino se presentan mayores niveles de ansiedad, acentúandose entre los mexico-norteamericanos. Los autores finalizan indicandola necesidad de efectuar más estudios sobre el tema.
\end{abstract}

\section{ABSTRACT}

A survey was applied to three groups of college students (Mexican, Chicano and American) on both sides of the U.S.A.-Mexico border trying to measure assertiveness, which is a response to anxiety-causing situations and consists of a defense of personal rights but keeping a respectful attitude toward the rights of others.

Results show a high anxiety level and a noticeable decrease of assertiveness among the Chicano, and for the three groups, females show higher anxiety levels, even higher in the Mexican-American group. Finally, authors establish the need for more studies on this subject.

\section{INTRODUCCIÓN}

La vecindad entre dos países siempre presenta un gran número de facetas interesantes de estudiar y explicar; no todas ellas son siempre positivas o negativas, pero ofrecen un vasto campo de acción para los estudiosos de los fenómenos sociales.

\footnotetext{
- Ángel Manuel Ramirez Peredo, licenciado en psicología, profesor asociado en la Escuela de Ciencias de la Educación de la UaBC.

** Teresa Mange Cabrillas, técnico académico en la Escuela de Ciencias de la Educación de la UABC
} 
Dentro de la psicología, este campo ha sido abordado mediante las investigaciones transculturales, que en México han tenido como principal enfoque la contrastación de la personalidad entre mexicanos y norteamericanos, dada la inmediata vecindad entre ambos países. En este rubro destacan los trabajos del Dr. Rogelio Díaz-Guerrero y - desde una perspectiva diferente- la labor del Dr. Santiago Ramírez. Estos autores han intentado definir la personalidad del mexicano desde un enfoque psicológico, a diferencia de otras aproximaciones de tipo sociológico o cultural (Paz, 1959, entre otros).

Díaz-Guerrero (1982) realizó un estudio acerca de las premisas socioculturales del mexicano, y propuso una dicotomía que denomino "activopasivo". Con ella explica un tipo particular de comportamiento característico en la forma de enfrentar los sucesos de la vida cotidiana. Sostiene este autor que los ciudadanos de origen norteamericano se enfrentan a los acontecimientos de la vida en una forma activa, es decir, superan los problemas que se les presentan modificando el ambiente. En contraposición, los mexicanos se conducen de manera pasiva, o sea, no modifican el medio, sino que se modifican a sí mismos. Pero, ¿qué pasa con los chicanos? Con base en las afirmaciones anteriores, surge la pregunta sobre la situación psicosociocultural de estos individuos que se encuentran bajo la presión de mantener un sistema conceptual propio y adoptar uno cualitativamente distinto, circunstancia que incide en la ansiedad que puede llegar a ocasionar y, por ende, en la manera de manejar esa situación estresante.

Por otro lado, un lugar importante en el campo de la psicología aplicada es ocupado por el interés en la evaluación y la eliminación de la ansiedad, provocada por el ritmo de vida que demanda la sociedad contemporánea (Coleman, Butcher y Carson, 1988), circunstancia que en ocasiones recibe el nombre de estrés. Uno de los factores que influyen en el desarrollo de la ansiedad, según algunos autores (Bartolomé, Carrobles, Costa y Del Ser, 1976), es la formaen que el individuo se relaciona con sus semejantes y puede manifestar sus opiniones o defender sus derechos. Esta manifestación puede asumir tres patrones característicos: agresividad (se ofende y atenta contra los derechos de los demás); pasividad (se acepta que los demás tienen más derechos que él); o asertividad (se defienden los derechos personales pero respetando los de los demás, no se pasa encima de ellos).

En este estudio se plantea que la ansiedad presente en los estudiantes de la Universidad Autónoma de Baja California (UABC) y de la Universidad Estatal de San Diego (SDSU), está relacionada en forma inversa con el grado de asertividad con que estos sujetos se conducen cotidianamente. Para poder encontrar tal relación, se emplearon dos instrumentos reconocidos, como son el Inventario de Ansiedad Rasgo-Estado (IDARE en cspañol, 
Spielberger y Díaz-Guerrero, 1975; STAI en inglés, Spielberger, 1980) y la Escala de Asertividad de Rathus (Rathus, 1973).

La relevancia de los resultados obtenidos radica en el hecho de que arrojan luces acerca de la poca veracidad de algunas conceptualizaciones hechas sobre la personalidad del mexicano, principalmente en el sentido de ser "pasivos", por lo menos no ocurre así en los sujetos que estudiamos. Por otra parte, señalan la necesidad de estudiar más profundamente la problemática de los mexicanos nacidos en los Estados Unidos, principalmente en relación con las consecuencias de carácter psicológico a raíz de su condición social particular.

\section{MARCO DE REFERENCIA}

El presente trabajo es resultado de una investigación que surgió como un esfuerzo para contribuir al análisis de los factores que agobian al habitante fronterizo, específicamente los estudiantes universitarios, así como sus expectativas de vida. El estudio de éstos nos permitirá en un futuro tomar medidas y establecer estrategias para disminuir los niveles de ansiedad.

Por ello, es preciso apuntar que las situaciones estresantes en las que se encuentra inmersa esta población tienden a disminuir su nivel de atención y concentración, afectando de manera negativa sus capacidades intelectuales y cognocitivas (Lazarus, 1977; Sharma, Dang y Spielberger, 1986), repercutiendo en el aprendizaje de los diversos contenidos manejados durante su vida estudiantil.

Para nuestro interés, cuando hablamos de ansiedad es necesario establecer una relación entre este concepto y el de asertividad, pero, ¿qué relación existe entre ambos? Hasta donde se sabe, tal relación es de tipo inverso, puesto que cuando una persona se enfrenta constantemente a situaciones provocadoras de ansiedad, el organismo emite algún tipo de respuesta con el fin de mantener el equilibrio, dichas respuestas pueden ser de carácter asertivo, agresivo o pasivo (McKay, Davis y Fanning, 1985). Díaz-Guerrero (1982) propone que es imposible hacer una separación entre estos conceptos, ya que la presencia de uno implica necesariamente la del otro.

Para un mejor conocimiento y comprensión del fenómeno, diremos que asertividad es el conjunto de comportamientos por los cuales el sujeto demanda respeto por sus derechos personales y expresa sentimientos, pensamientos y creencias de forma directa, honesta y apropiada, de suerte tal que no viola los derechos de otras personas.

Por otra parte, el concepto ansiedad se refiere a las conductas de tipo emocional, caracterizadas por alteraciones fisiológicas y psicológicas que 
se manifiestan ante la presencia de estímulos o circunstancias que alteran el esquema conceptual, emotivo o psicológico de una persona.

Otro concepto pertinente es el de estrés, por el cual se hace referencia a la tensión emocional excesiva, resultado de un desequilibrio entre las demandas del entorno y la capacidad del individuo para satisfacerlas. Este descquilibrio va a dar lugar a la ocurrencia de respuestas de ansiedad, es decir, el estrés es un proceso en el cual el desequilibrio va a provocar ansiedad, misma que deberá ser resuelta de alguna manera por el individuo sujeto de esa tension.

A partir de los planteamientos de Díaz-Guerrero, y tomando como base sus diversos estudios transculturales entre México y Estados Unidos, es posible inferir que los comportamientos de las dos sociedades van a producir diferentes niveles de ansiedad en los sujetos. A la luz de tales proposiciones teóricas, tal vez no sea muy arbitrario afirmar que las personas originarias de Estados Unidos, que han vivido toda o la mayor parte de su vida bajo el amparo de esa cultura y la han hecho parte de su idiosincracia, tienden hacia comportamientos más asertivos, lo cual hace que enfrenten de manera activa los diversos factores estresantes de la vida cotidiana, mecanismo mediante el cual climinan la ansicdad y evitan que ésta se acumule.

Por otra parte, los sujetos nacidos y criados en la cultura mexicana tienen mayor posibilidad de presentar altos niveles de ansiedad, ya que al ser menos asertivos y enfrentar los acontecimientos cotidianos de manera pasiva (Díaz-Guerrero, 1982), se produce un acumulamiento de ansiedad, que en algunos casos amenaza seriamente con alterar la salud mental de un elevado número de miembros de la población mexicana.

Consideramos que, para una mejor comprensión del fenómeno, es necesario profundizar un poco sobre el concepto de las premisas socioculturales propuesto por Díaz-Guerrero, así como su relación e influencias que tienen sobre los niveles de ansiedad y los comportamientos asertivos de las personas.

Pero ¿qué son las premisas socioculturales? Cuando hablamos de éstas nos referimos a las afirmaciones, ideas, pensamientos y reglas que de manera implícita o explícita son utilizadas y aprobadas consistentemente por la mayoría de los miembros de una cultura en particular (Díaz-Guerrero, 1982), y que rigen y determinan las conductas a seguir de cada uno de sus miembros.

Ahora, ¿qué relación hay entre estas ideas generalizadas y la asertividad-ansiedad, y cómo interactúan una con otra?

En primer lugar, es pertinente mencionar los postulados que manejan los profesionales de la terapia racional emotiva (Ellis, 1957; Beck, 1976; 
Bandura, 1975; Lazarus, 1977), quienes plantean que existen tres formas de comportamientos manifestados por los sujetos en su relación cotidiana con el medio; dichos comportamientos pueden estar guiados por la agresividad, la asertividad y la no asertividad, también llamada pasividad (McKay, Davis y Fanning, 1985).

Los comportamientos agresivos se caracterizan por ser aquéllos en los cuales los sujetos imponen sus derechos personales por encima de los demás, sin importarles los efectos que dichas conductas puedan producir en las otras personas.

Por su parte, los comportamientos asertivos son aquéllos en los que el sujeto defiende sus derechos personales, exige respeto hacia su persona, pero no olvida los derechos que los demás sujetos tienen para ser respetados, lo cual le permite expresar sus pensamientos en forma directa y abierta.

Finalmente, los comportamientos no asertivos caracterizan a las personas que no defienden sus derechos, permiten que se les pisotee, subestime y denigre su calidad humana y sus derechos personales. Es cuando los sujetos niegan la importancia de sus derechos, y en cambio conceden una gran importancia a las ideas y pensamientos de los demás.

Díaz-Guerrero se ha interesado por estudiar y comparar más de cerca la problemática que presentan los habitantes de diferentes países y culturas. Con las múltiples investigaciones realizadas en el plano transcultural, Díaz-Guerrero (1982) se ha propuesto evaluar las diferencias ideológicas, psicológicas y de personalidad, que él llama "filosofía de vida", entre los habitantes de distintas culturas, niveles socioeconómicos y socioculturales. Él establece diferencias significativas en las formas de enfrentar el estrés (dicotomía activo-pasivo) en el tipo de personalidad y en las relaciones interpersonales, considerando que dichos estudios se refieren a poblaciones de las regiones céntricas del país (Ciudad de México, en particular), especialmente de las clases socioeconómicas bajas.

Ante esta situación resulta de primordial importancia establecer líneas de investigación que cuestionen la validez de esos resultados generalizados a los habitantes de las franjas fronterizas, específicamente de ambas Californias, y —en el caso que nos ocupa- a una población compuesta por estudiantes universitarios de un nivel socioeconómico y cultural diferente a las muestras utilizadas por ese autor.

Es evidente que nuestra población, por su ubicación geográfica y nivel sociocultural, difiere grandemente de las del centro del país, puesto que el hecho de vivir en una zona donde hay diferentes formas de pensamiento, conformadas e influenciadas por las premisas culturales propias de los habitantes del otro lado de la frontera, puede llegar a producir un desequi- 
librio emocional en el sujeto, ya que se presenta un sentimiento de no pertenencia cultural, posible favorecedor de un aumento en los niveles de ansiedad de esa población.

Esta circunstancia permite hablar de una problemática propia de la zona fronteriza, intimamente relacionada con los dos conceptos centrales de nuestra investigación (asertividad-ansiedad).

Retomando el planteamiento de Díaz-Guerrero, en el que la dicotomía activo-pasivo es un factor decisivo en el nivel de ansiedad, es factible suponer que este nivel puede incrementarse en esta zona fronteriza donde se ha operado un cambio cultural; es una creencia generalizada que las personas -en esta zona - no piensan tan pasivamente, donde los roles sociales son diferentes, al igual que son diferentes y mayores las exigencias hacia la gente recién inmigrada, en su gran mayoría formada por sujetos que provienen de otros estados de la república y cuyas premisas son diferentes, dado que en ocasiones aún no están preparados psicológicamente para enfrentar de manera activa las situaciones estresantes de la vida cotidiana en esta zona del país.

Por otra parte, los sujetos de la cultura norteamericana tienen una visión y una forma de enfrentar la vida totalmente distinta a la que utiliza el mexicano. Los norteamericanos enfrentan la vida y las situaciones estresantes de manera activa, tratan de enfrentar las situaciones embarazosas, no las evitan, las encaran, y al salir airosos de ellas, se refuerza y fortalece su seguridad y el nivel de asertividad, mientras que los mexicanos prefieren hacer todo lo posible por evitar las situaciones molestas aplicando frecuentemente su viejo legado popular: No hagas hoy lo que puedas dejar para mañana (Diaz-Guerrero, 1982:155), lo que puede producir un incremento de la ansiedad y un decremento en la posibilidad de actuar asertivamente.

Por lo anteriormente citado, en este estudio piloto planteamos la hipótesis de que los miembros de la cultura estadounidense presentan mayor grado de asertividad y menor de ansiedad; mientras que los mexicanos tienden hacia un menor grado de asertividad y uno elevado de ansiedad.

Un sector importante dentro de ambas poblaciones, característica distintiva de las franjas fronterizas, se refiere al grupo integrado por sujetos que comparten rasgos de ambas culturas, es decir, personas que tienen su origen en un país pero que han crecido bajo rasgos culturales diferentes, propios de otra nación. En el caso que nos ocupa, hablamos de ciudadanos de origen mexicano conocidos tradicionalmente como chicanos, designación que emplearemos a lo largo de este informe para referirnos a sujetos con antecedentes familiares mexicanos pero que han crecido en el seno de la sociedad estadounidense. Pensamos que este sector de la población 
presenta características de personalidad específicas dado el conflicto creado por el choque cultural dentro del cual se encuentran inmersos.

La importancia de abocarnos al estudio del grupo chicano estriba en que, son una minoría étnica dentro de la sociedad estadounidense, en la cual se les dificulta mantener su cultura, su herencia y su historia propia, y son sujetos de fuerte discriminación racial, social, económica y cultural. Villanueva (1985) afirma que las disciplinas académicas prestaban poca o ninguna atención a la investigación del pueblo chicano, y los pocos trabajos realizados adolecían de prejuicios y de una mínima participación de este grupo social.

En su obra, Villanueva (1985) hace una reseña de la literatura que aborda la problemática chicana, señalando que en no pocos casos el investigador considera a ese grupo como inferior, con una gran necesidad de incorporarlo al sistema de vida americano. Esta circunstancia ha dado lugar a grandes diferencias entre distintas generaciones de familias méxico-americanas (intergeneraciones) y las mexicanas, en aspectos tales como el sistema de valores y creencias, estilos de confrontación, percepción de la economía y aspiraciones hacia el futuro, así como en la flexibilidad cognoscitiva.

En general, resulta evidente que el pueblo chicano debe ser comprendido desde su propia perspectiva histórica, en la que la explotación, la discriminación, la ignorancia de sus tradiciones y el afán de borrar su cultura han sido constantes a lo largo de su existencia.

Por lo expuesto en párrafos anteriores, nuestra investigación se basa en las relaciones existentes entre estos dos conceptos en estudiantes universitarios mexicanos, norteamericanos (origen sajón) y chicanos, la cual permitirá confirmar o rechazar la hipótesis planteada respecto a la relación propuesta entre asertividad y ansiedad, concretamente en el tipo de sujetos empleados.

\section{METODOLOGÍA}

Para la realización de este estudio se utilizó una muestra integrada por 167 estudiantes universitarios no graduados, los cuales se dividieron en tres grupos: 57 alumnos de la Universidad Autónoma de Baja California ( 26 femeninos, 31 masculinos) en la unidad Mexicali, 66 del campus de la Universidad Estatal de San Diego (SDSU) en esa ciudad (30 femeninos, 36 masculinos) y 44 del campus de Caléxico ( 21 femeninos, 23 masculinos).

Ambos grupos fueron elegidos de manera aleatoria, sin considerar el semestre ni la carrera que actualmente cursaban. No se controló el nivel 
socioeconómico. La variable sexo se intentó mantener equilibrada en los dos grupos, presentándose pequefias diferencias en las proporciones de la misma en cada uno de ellos.

Las edades de los estudiantes fluctuaron de manera equitativa en los tres grupos; el formado por sujetos radicados en Estados Unidos de origen mexicano (chicano) tuvo una media (que en adelante se representará con la letra M) de 20.8 afios de edad; los estudiantes de origen estadounidense, alcanzaron una media de 20.9 affos; por otra parte, la media de edad del grupo mexicano fue de 21.3 años.

Resulta conveniente sefalar que este trabajo se considera como un estudio piloto, con el cual se obtendrán datos que sirvan de orientación para las futuras investigaciones que se realicen en mayor escala.

Para obtener la información necesaria se utilizaron dos instrumentos, la Escala de Asertividad de Rathus (RAS), conformada de 30 reactivos, los cuales de manera gradual indican el nivel de asertividad en los sujetos. Las posibles respuestas van en una escala de $-3 \mathrm{a}+3$, mismas que se suman para obtener una calificación total. En algunos reactivos el signo de la respuesta se invierte, pues pretenden controlar una tendencia en los sujetos a contestar en cierto sentido únicamente.

Una calificación cercana a +3 indica un elevado grado de asertividad, en tanto que -3 señala un bajísimo nivel de este rasgo; el valor intermedio es de 0 (cero) y corresponde a un nivel deficiente. Una característica importante de este instrumento es que sus calificaciones no son unidades estándares, limitante que buscamos superar mediante la transformación de los datos crudos en unidades $\mathrm{T}(\mathrm{M}=50, \mathrm{DS}=10)$ y así comprobar estadísticamente las diferencias entre estas medidas y las del IDARE. A pesar de las restricciones de representatividad que conlleva esta circunstancia, los resultados nos indicarán la tendencia en la respuesta de los sujetos.

Esta escala se obtuvo de la versión original en inglés, publicada en la revista Behavior Terapy (1973). La versión en español se tomó de Práctica de la Terapia de la Conducta (Bartolomé y cols., 1976). Una de sus ventajas es que pucde ser empleada con fines de práctica profesional, así como en investigación clínica.

El autor de la RAS informa que la confiabilidad se obtuvo mediante dos procedimientos distintos, test-retest y división por mitades; en el primero, la prueba se aplicó a 68 estudiantes universitarios con un intervalo de ocho semanas entre ambas administraciones, y se calculó un coeficiente de correlación producto-momento de Pearson que arrojó una $\mathrm{r}=0.7782$ ( $p<0.01)$, que indica una alta estabilidad entre las calificaciones obtenidas; en el segundo procedimiento, se emple 6 una división por reactivos paresnones de los instrumentos aplicados a 67 personas no universitarias, se 
calculó el coeficiente de correlación mencionado anteriormente, obteniéndose una $r=0.7723$ significativa a nivel $p<0.01$ (Rathus, 1973).

Por lo que se refiere a la evaluación de validez, ésta se obtuvo en relación con dos criterios externos; uno fue la calificación que obtuvieron los sujetos empleados para la confiabilidad por pares-nones en una escala tipo diferencial semántico, formada por 17 adjetivos bipolares y contestada por personas que conocían muy bien a los respondientes; se determino la estructura factorial de la escala, identificándose cuatro factores que explicaban el $71.2 \%$ de la varianza total: asertividad, satisfaccion, inteligencia y prosperidad, y salud; además se correlacionaron las calificaciones en la prueba de Rathus con cada una de las escalas de diferencial semántico, encontrando valores significativos $(p<0.01)$ con seis de ellas, cinco en sentido positivo y uno negativo, que le permite afirmar a su autor que la prueba es un indicador válido de la asertividad en términos de las impresiones que los demás tienen de un sujeto y no como un deseo de contestar en una forma aceptada socialmente. El segundo criterio empleado fueron las calificaciones dadas a entrevistas audiogra badas por jueces imparciales (cuya confiabilidad fue de $\mathrm{r}=0.9382$ ) correlacionadas con las puntuaciones de la prueba, arrojando una $\mathrm{r}=0.7049$ con $\mathrm{p}<0.01$ (Rathus, 1973).

Además, este autor encontró que 27 de los 30 reactivos correlacionaban significativamente con la calificación total, 23 a nivel de $p<0.01$ y 4 con una $p<0.03$; el rango de los valores iba desde $r=.2362$ hasta $r=0.7006$ (Rathus, 1973).

Otros investigadores (Pál-Hegedus y Jensen, 1982) encontraron que la confiabilidad de este instrumento, empleando el coeficiente de Cronbach, alcanz 6 un valor igual a 0.83 , lo que confirma su elevada consistencia interna. A diferencia del creador del instrumento, encontraron que la varianza total de la prueba podía ser explicada mediante tres factores: ansiedad social, confrontaciones en situaciones de consumo o servicio y libre expresión de emociones u opiniones.

Para medir la variable ansiedad, se utilizó el inventario de ansiedad rasgo-estado (IDARE), de Charles Spielberger y R. Díaz- Guerrero (1975), en su versión española, y la versión en inglés del mismo instrumento(STAI) de Spielberger (1987).

Las escalas del IDARE se componen de 40 reactivos, los cuales permiten obtener una visión global de la ansiedad, ya sea como un rasgo característico de la personalidad de los sujetos (ansiedad-rasgo), o como un estado temporal provocado por una situación particular presente al momento de la aplicación del instrumento (ansiedad-estado).

Las unidades de calificación son puntuaciones T; de esta manera, los niveles elevados de ansiedad reciben puntuaciones por arriba de 50 . Las 
medidas de confiabilidad se obtuvieron mediante el método de test-retest con intervalos de 1 hora, 20 y 104 días; para la subprueba deansiedad-rasgo se alcanzaron valores en varones de $r=0.84,0.86$ y 0.73 respectivamente; en mujeres, los resultados fueron de $\mathrm{r}=0.76,0.76$ y 0.77 .

En la medida de ansiedad-estado, la confiabilidad tuvo las siguientes magnitudes: en varones, la $\mathrm{r}=0.33,0.54$ y 0.33 ; para mujeres, $\mathrm{r}=0.16,0.27$ y 0.31. Las correlaciones bajas para la medida de ansiedad-estado fueron anticipadas, puesto que una medida válida de esta dimensión debe reflejar la presencia de factores situacionales únicos presentes al momento del examen.

Para validar la escala de ansiedad-rasgo se utilizó el método de validez concurrente, relacionándola con pruebas como el IPAT y la escala de ansiedad manifiesta (TMAS), con ambas se obtuvieron correlaciones superiores a $\mathrm{r}=\mathbf{0 . 7 5}$, tanto con mujeres como con varones universitarios. Por lo que respecta a la subprueba de ansiedad-estado, se empleó una muestra de 977 estudiantes universitarios bajo dos condiciones experimentales, una estándar (norma), y otra pidiéndoles que se sintieran como si estuvieran a punto de presentar "la prueba final de uno de los cursos más importantes" (examen). Los resultados fueron calificaciones más altas, en promedio, bajo esta situación que en la norma, tanto para varones (54.99 vs. 40.02) como para mujeres (60.51 vs. 39.36).

Es importante señalar que los cuestionarios aplicados presentaron una pequeña diferencia en cuanto al contenido; los aplicados a estudiantes estadounidenses, en su versión original inglesa, no inclúan información acerca del significado del concepto asertividad, mientras que los aplicados a los mexicanos sí la contenían. Consideramos necesario mencionar este detalle, porque es probable que los resultados obtenidos puedan haber sido influidos por esta variable, lo que se discutirá posteriormente.

Una vez seleccionada la muestra, se aplicaron los instrumentos previamente mencionados; 66 fueron aplicados en el campus de San Diego (a sujetos de habla inglesa), 44 a estudiantes de origen mexicano residentes en Estados Unidos (chicanos); y 57 a estudiantes de la UABC. Para los estudiantes del campus de la Universidad Autónoma de Baja California y de Caléxico, la aplicación de los dos instrumentos se realizó en forma simultánea, mientras que para los de San Diego se aplicó primeramente la escala de asertividad de Rathus, y posteriormente les fueron enviados por correo los cuestionarios de STAI, los cuales ya contestados fueron devueltos por el mismo medio.

Para obtener las puntuaciones $T$ de los cuestionarios del IDARE se utilizaron las tablas de baremos adaptadas para la población mexicana por Spielberger y Díaz-Guerrero; mientras que para los estudiantes norteamericanos se utilizó la versión original en inglés, elaborada por $\mathrm{Ch}$. Spielberger. 
El análisis estadístico de los datos se efectuó mediante el programa SPSS PC+3.0, con el cual se evaluaron las calificaciones obtenidas por los sujetos en las dos variables (asertividad y ansiedad, en sus variantes estado y rasgo), tomando en consideración -como variables independientes-el sexo y la nacionalidad (identificada por la universidad de pertenencia).

\section{DESCRIPCIÓN E INTERPRETACIÓN DE RESULTADOS}

Los resultados globales obtenidos de la aplicación de los instrumentos se presentan en forma resumida en el cuadro 1. En ella se muestran las medias totales en asertividad, ansiedad-estado y ansiedad-rasgo para cada uno de los subgrupos (chicanos, americanos y mexicanos). Como puede apreciarse, en la variable asertividad los mexicanos obtuvieron las calificaciones más elevadas $(M=50.00)$, seguidas por los chicanos $(M=49.9773)$ y por los norteamericanos $(M=49.7727)$. Las diferencias entre grupos se probaron mediante el estadístico $\mathrm{F}$ de Fisher, sin que alcanzaran un nivel aceptable de significancia.

En la variable ansiedad-estado, el ordenamiento se cambia y la posición más alta la ocupa el grupo chicano $(M=51.0455)$, mientras que la población mexicana obtuvo $(M=46.2982)$ y los estadounidenses tuvieron la posición intermedia (M=47.0303). En este caso, las diferencias, evaluadas con la prueba $F$, tampoco alcanzaron un nivel aceptable de significancia.

Por último, en ansiedad-rasgo, nuevamente los chicanos obtuvieron la calificación más alta $(M=50.8864)$, seguidos por los mexicanos $(M=43.5789)$ y los norteamericanos en la posición intermedia $(M=43.4848)$. Al emplear un análisis de varianza, se obtuvo una $\mathrm{F}=2.8308$ con una significancia de $\mathrm{p}<0.0619$.

CUADRO 1. Población por variables.

\begin{tabular}{ccccccc}
\hline Grupo & \multicolumn{2}{c}{ Asertividad } & \multicolumn{3}{c}{ Ansiedad-Estado } & \multicolumn{2}{c}{ Ansiedad-Rasgo } \\
& M & DS & M & DS & M & DS \\
\hline
\end{tabular}

$\begin{array}{lrllrrr}\text { Chicanos } & 49.9773 & 10.1672 & 51.0455 & 7.7338 & 50.8864 & 10.9613 \\ \text { Norteam. } & 49.7727 & 10.9962 & 47.0303 & 19.9769 & 43.4848 & 19.7439 \\ \text { Mexicanos } & 50.0000 & 10.0623 & 46.2982 & 16.9821 & 43.5789 & 19.0516 \\ \text { Total } & 49.9042 & 10.4063 & 47.8383 & 16.5181 & 45.4671 & 17.7983\end{array}$

NOTA: Diferencias expresadas en medias y desviaciones estándares. 


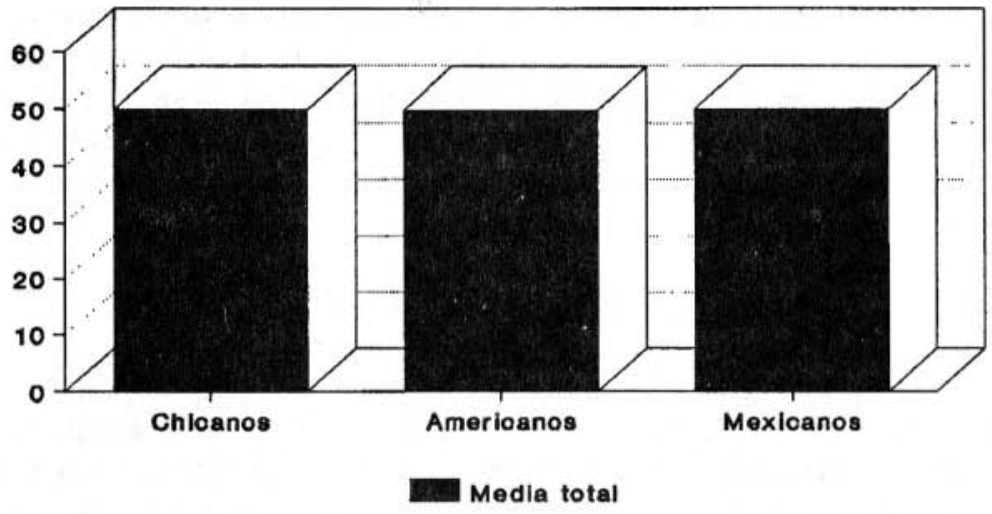

FIGURA 1. Asertividad. Medias de población general.

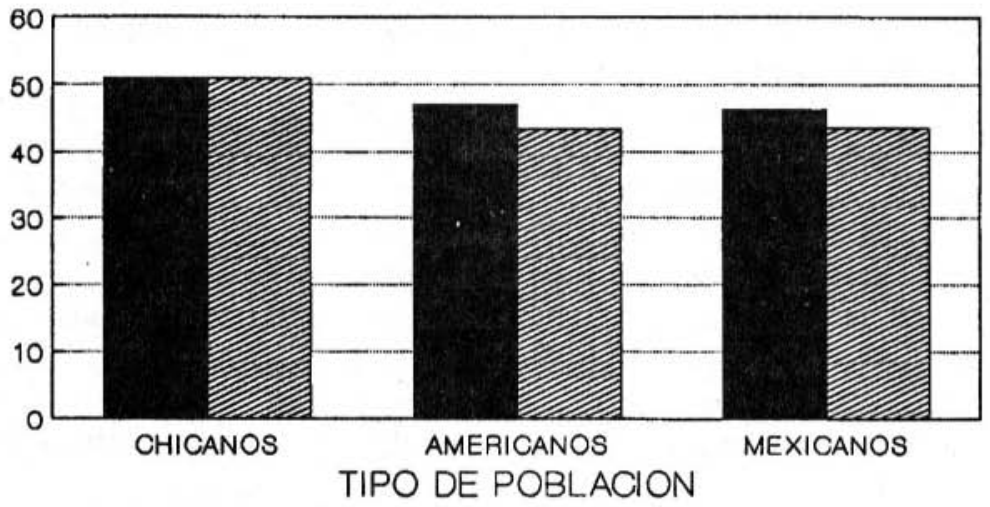

ansiedad ESTADO ANSIEDAD RASGO

FIGURA 2. Ansiedad. Media de población general.

\section{COMPARACIÓN ENTRE SEXOS}

En primer lugar, en asertividad se observa que los hombres tienen una calificación más alta $(\mathrm{M}=50.9667)$ que las mujeres $(\mathrm{M}=48.6683)$, la diferencia tuvo un valor de $\mathrm{F}=2.0476$, con un nivel de significancia de $\mathrm{p}<0.15$ (ver cuadro 2).

En el subgrupo sexo femenino, las integrantes chicanas presentan la puntuación más baja en asertividad (M=48.0952), mientras que las nortea- 
CUADRO 2. Asertividad por sexo y población.

\begin{tabular}{lllll}
\hline Grupo & \multicolumn{2}{c}{ Femenino } & \multicolumn{2}{c}{ Masculino } \\
& M & DS & M & DS \\
\hline Chicanos & 48.0952 & 11.640 & 51.6957 & 8.5090 \\
Norteamericanos & 49.500 & 12.5856 & 50.000 & 9.6540 \\
Mexicanos & 48.1538 & 10.9643 & 51.5484 & 9.1318 \\
Total & 48.6623 & 11.6660 & 50.9667 & 9.1264 \\
& & & & \\
\hline
\end{tabular}

NOTA: Diferencias expresadas en medias y desviaciones estándares.

mericanas alcanzaron la calificación más alta $(\mathrm{M}=49.50)$ y las mexicanas la posición intermedia $(M=48.1538)$. Tales diferencias no fueron estadísticamente significativas.

En el subgrupo sexo masculino, la situación es completamente diferente, ya que los chicanos ocupan la posición más elevada $(M=51.6957)$ y los americanos la inferior $(M=50.00)$, mientras los mexicanos se encuentran entre ambos grupos ( $\mathrm{M}=51.5484)$. $\mathrm{Al}$ igual que en el caso femenino, las diferencias no fueron significativas.

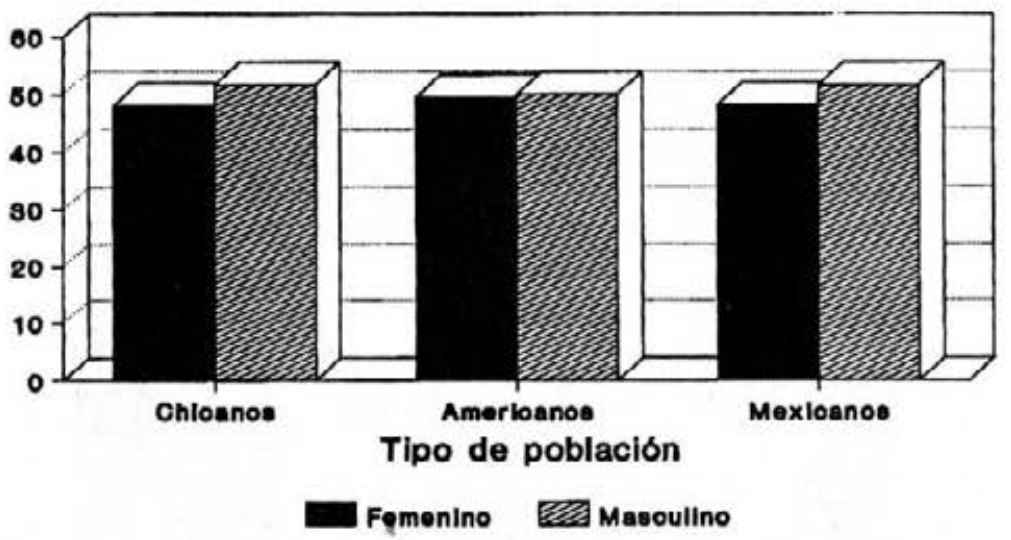

FIGURA 3. Asertividad. Medias por sexo y población.

Para la variable ansiedad-estado, el subgrupo femenino obtuvo una calificación mayor $(M=52.2727)$ que el masculino $(M=44.0444)$ lo que indica que las mujeres en general presentan una mayor ansiedad-estado que los hombres (ver cuadro 3). La prueba de $\mathrm{F}$ alcanzó un valor de 10.912 , con una significación de $p<0.001$. 
CUADRO 3. Ansiedad-estado por sexo y población.

\begin{tabular}{lrrrr}
\hline Grupo & \multicolumn{2}{c}{ Femenino } & \multicolumn{2}{c}{ Masculino } \\
& M & \multicolumn{1}{c}{ DS } & M & \multicolumn{1}{c}{ DS } \\
\hline Chicanos & 50.8095 & 8.1094 & 51.2609 & 7.5511 \\
Norteamericanos & 53.8667 & 11.0289 & 41.3333 & 23.8100 \\
Mexicanos & 51.6154 & 10.4846 & 41.8387 & 20.0418 \\
Total & 52.2727 & 10.0808 & 44.0444 & 19.7626 \\
& & & & \\
\hline
\end{tabular}

NOTA: Diferencias expresadas en medias y desviaciones estándares.

Dentro del subgrupo femenino, encontramos que las norteamericanas tienen la calificación más alta $(M=53.8667)$ en tanto que las chicanas la más baja ( $M=50.8095)$, en medio se ubican las mexicanas $(M=51.6154)$. No hubo un nivel aceptable de significancia estadística.

En el caso de los hombres, la posición de los chicanos es totalmente diferente, puesto que su calificación promedio es la más elevada $(M=51.2609)$, lade los mexicanos es intermedia ( $M=41.8387)$ y los americanos la inferior $(\mathrm{M}=41.3333)$. A diferencia del caso anterior, la diferencia fue significativa con una $\mathrm{p}=0.07$.

En lo referente a la variable ansiedad-rasgo, nuevamente el subgrupo femenino obtuvo una calificación superior $(M=48.6104)$ a la del masculino $(\mathrm{M}=42.7778)$. Las prueba $F$ mostró una significancia de $\mathrm{p}=0.034$ ( $\mathrm{F}=4.5518)$.

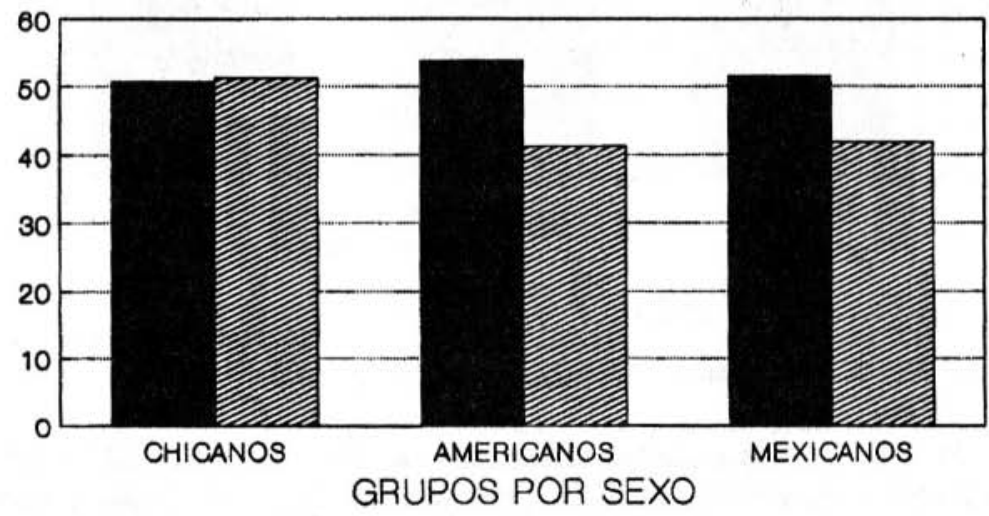

FEMENINO $\mathbb{Z}$ MASCULINO

FIGURA 4. Ansiedad estado. Medias de población por sexo. 
CUADRO 4. Ansiedad-rasgo por sexo y población.

\begin{tabular}{lrrrr}
\hline Grupo & \multicolumn{2}{c}{ Femenino } & \multicolumn{2}{c}{ Masculino } \\
& M & \multicolumn{1}{c}{ DS } & M & DS \\
\hline Chicanos & 52.4762 & 8.3583 & 49.4348 & 12.9117 \\
Norteamericanos & 46.0333 & 12.5134 & 41.3611 & 24.1663 \\
Mexicanos & 48.4615 & 14.6948 & 39.4839 & 21.4381 \\
Total & 48.6104 & 12.4870 & 42.7778 & 21.0199 \\
& & & & \\
\hline
\end{tabular}

NOTA: Diferencias expresadas en medias y desviaciones estándares.

En el subgrupo femenino, las chicanas ocupan el lugar más alto $(\mathrm{M}=52.4762)$, seguidas de las mexicanas $(\mathrm{M}=48.4615)$ y de las norteamericanas $(M=46.0333)$. De nueva cuenta, la significación estadística a $\mathrm{p}=0.21$ (ver cuadro 4).

Para el subgrupo masculino, tenemos que los chicanos presentan mayor ansiedad-rasgo $(M=49.4348)$, seguidos por los norteamericanos $(M=41.3611)$ y los mexicanos $(M=39.4839)$. Estas diferencias fueron significativas a nivel de $\mathrm{p}=0.13$.

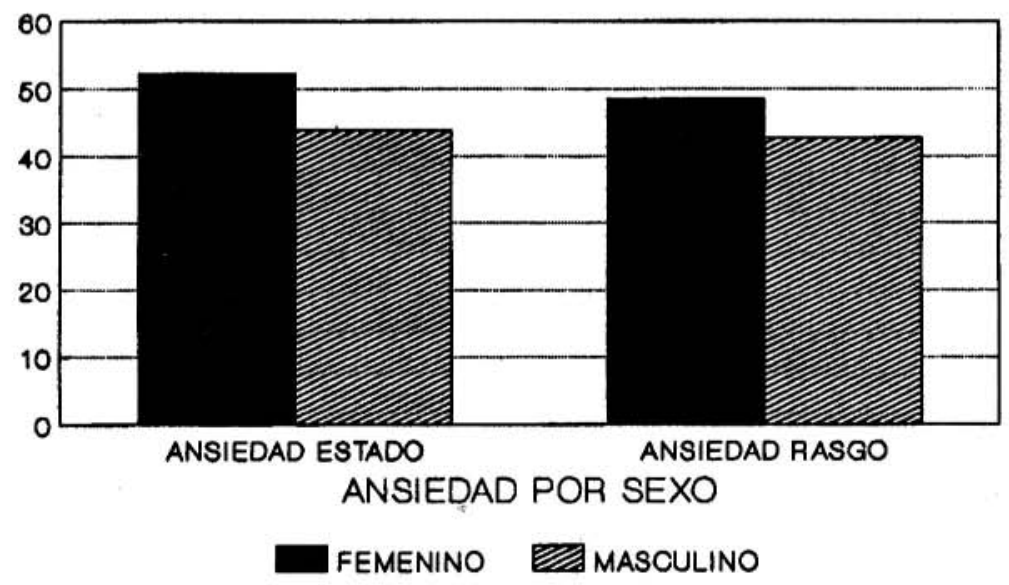

FIGURA 5. Ansiedad. Medias por sexo.

\section{ASOCIACIONES ENTRE INSTRUMENTOS}

Para analizar la relación que existe entre las medidas arrojadas por los instrumentos empleados, se utilizó el coeficiente de correlación de Pearson 
(r). Los resultados señalan que la asociación entre las medidas de asertividad y las dos de ansiedad son muy bajas, casi nulas, con ansiedad-estado la $r=-0.0814$; con ansiedad-rasgo, $r=-0.0739$. En este caso, es interesante seffalar que el signo confirma el sentido de la relación, si bien los valores obtenidos no son estadísticamente significativos.

Al relacionar los resultados de ansiedad-estado y ansiedad-rasgo, la asociación es en extremo elevada $(r=0.7458)$ con una significancia al nivel de $\mathrm{p}=0.001$, que no resulta sorprendente, pues aunque se trata de la misma variable, las modalidades son cualitativamente distintas, como se explic 6 anteriormente.

Los valores obtenidos en este análisis indican que las mediciones de las dos modalidades de ansiedad se hallan estrechamente vinculadas.

\section{INTERPRETACIÓN}

La información anteriormente descrita señala que, en términos globales y para el tipo de sujetos muestreados, existe un nivel moderado de ansiedad - tanto estado como rasgo - y uno ligeramente alto de asertividad (ver renglón de total en el cuadro 1). Es decir, considerando a los estudiantes como una población homogénea (si se toma en cuenta la condición académica que enfrentan a nivel universitario), no se presenta la asociación propuesta entre esas dos variables. Este resultado puede ser debido tanto a una baja relación existente entre los instrumentos empleados, como al pequefio número de individuos estudiado, situación que habría que evitar con una muestra de mayor escala.

Al hacer un examen más minucioso de las calificaciones intergrupales nos encontramos con el hecho de que la hipótesis planteada inicialmente sí se cumple parcialmente; esto es, si se comparan las puntuaciones de las mujeres y de los hombres en asertividad con las correspondientes en ansiedad-estado y rasgo, nos daremos cuenta que el grupo con baja calificación (femenino) en la primera variable obtendrá un valor elevado en las segundas. Este punto, debido a los niveles de significancia de las mediciones obtenidas, no es posible afirmarlo de manera categórica, sin embargo, la dirección de las calificaciones apuntan en ese sentido.

Dicho con otras palabras, el grupo femenino en su conjunto puntúa bajo en asertividad y -como se postuló inicialmente - califica alto en las mediciones de ansiedad en comparación con el masculino (ver cuadro 5) .

Un hecho importante a destacar es el que se refiere a la información proporcionada por las medidas de variabilidad (en este caso, desviación estándar). Como se observa en cada una de los cuadros mencionados, los distintos grupos se comportan de manera diferente. 
CUADRO 5. Variables por sexo

\begin{tabular}{lcccr}
\hline Grupo & \multicolumn{2}{c}{ Femenino } & \multicolumn{2}{c}{ Masculino } \\
& M & DS & M & \multicolumn{1}{c}{ DS } \\
\hline Asertividad & 48.6623 & 11.6660 & 50.9667 & 9.1264 \\
Asertiv.-Estado & 52.2727 & 10.0808 & 44.0444 & 19.7626 \\
Asertiv.-Rasgo & 48.6104 & 12.4870 & 42.7778 & 21.0199 \\
\hline
\end{tabular}

NOTA: Diferencias expresadas en medias y desviaciones estándares.

Si comparamos a los americanos con las otras muestras, encontramos que en asertividad, además de puntuar bajo, presentan una mayor variabilidad; es decir, que en el interior de este grupo los sujetos mantienen una mayor dispersión en la variable antes mencionada. Con base en esta información se puede afirmar que ese nivel bajo del grupo norteamericano es una característica muy homogénea entre sus integrantes, no así con los otros grupos (ver cuadro 1). Al respecto, seffalamos la conveniencia de realizar —con mayor detalle— un análisis mediante otros procedimientos estadísticos más refinados.

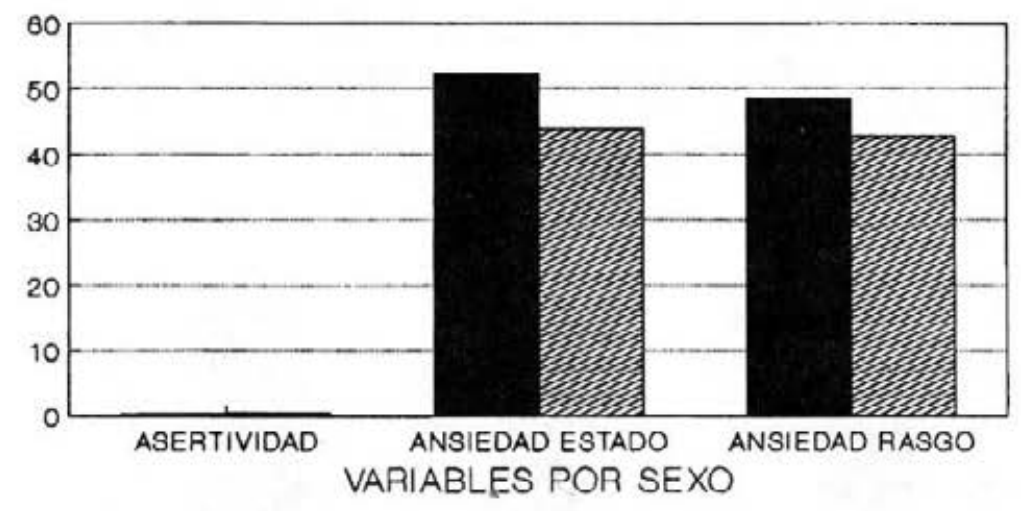

a femenino Masculino

FIGURA 6. Variables por sexo. Medias de variables por sexo.

Una deducción semejante es posible hacer en lo que respecta a la otra variable en estudio, ansiedad, en sus dos modalidades. En este caso, nuevamente el grupo chicano manifiesta niveles altos y más uniformes que los grupos de estadounidenses y mexicanos (ver cuadro 1). 


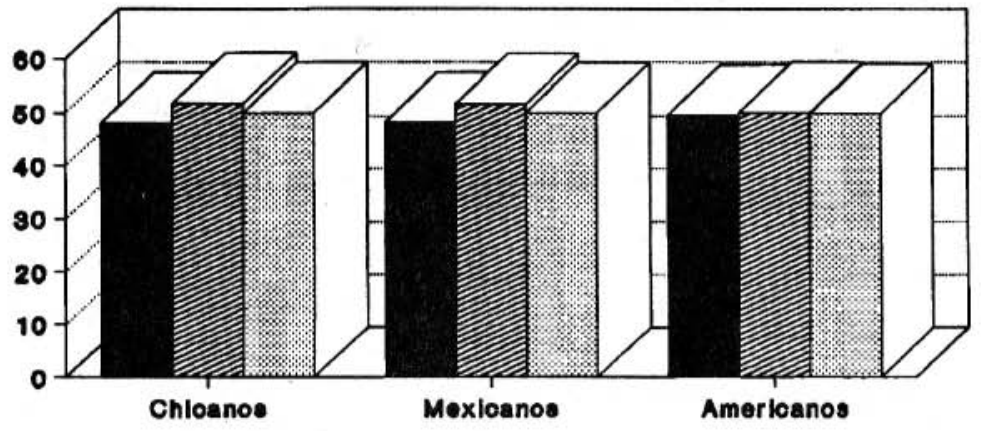

Femenino Media global

FIGURA 7. Asertividad. Variable por población y sexo.

Este hecho se presenta de manera distinta para el grupo de chicanos, tanto en las comparaciones hechas por sexo y ansiedad- estado, como por sexo y ansiedad-rasgo. A este respecto, el subgrupo masculino es el que muestra una uniformidad mayor que el femenino, tanto en asertividad, como en las dos modalidades de ansiedad.

Sin embargo, en las variables de ansiedad estado y ansiedad rasgo, son las mujeres estadounidenses y mexicanas las que presentan menor uniformidad en sus calificaciones, es decir, que en ellas no es una característica uniforme, mientras que en los grupos masculinos la variabilidad es notoriamente elevada.

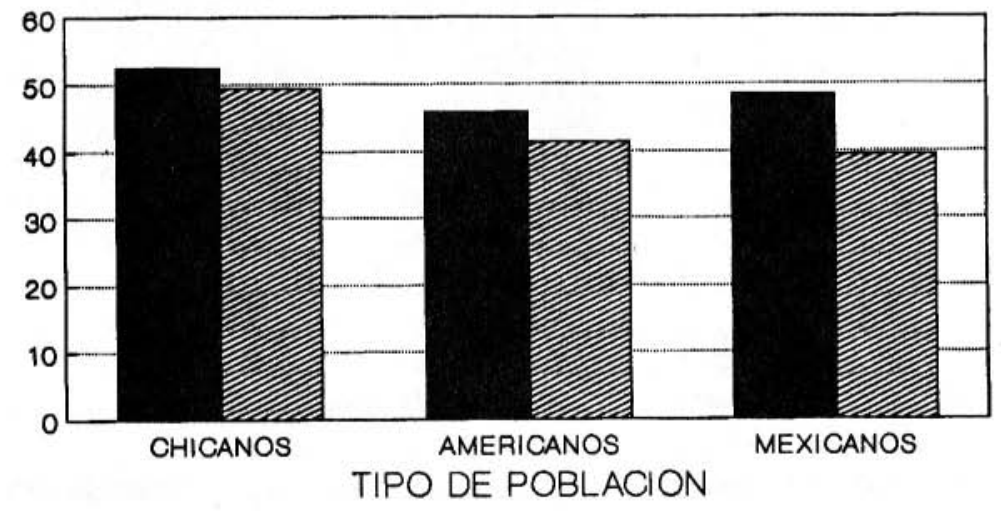

GEMENINO

FIGURA 8. Ansiedad rasgo. Medias por población y sexo. 


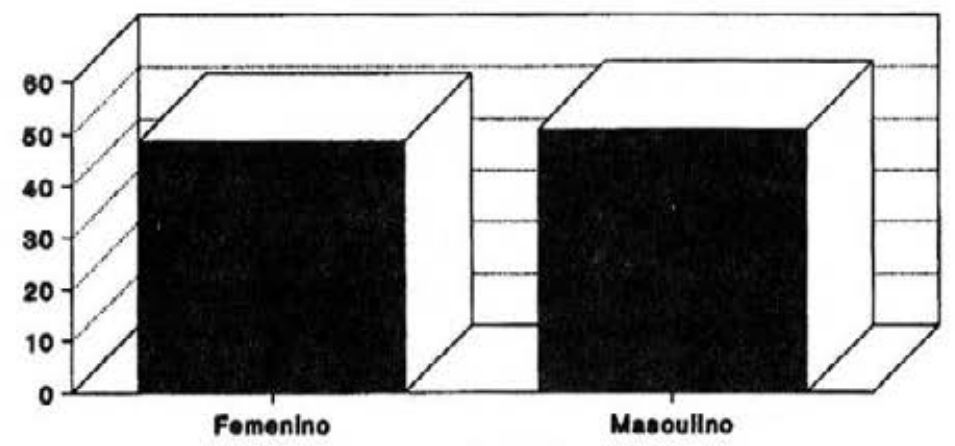

Asertividad por sexo

Media total

FIGURA 9. Asertividad. Medias totales por sexo.

Al comparar los grupos femenino y masculino (sin tomar en cuenta diferencias en nacionalidad) en cada una de las dos variables encontramos de nuevo que las mujeres tienen un menor nivel de asertividad que los hombres, pero una mayor variabilidad intragrupo. Por lo que toca a las variables de ansiedad, es el grupo femenino el que obtuvo las calificaciones más elevadas y la menor dispersión, si se le compara con los resultados del sexo masculino. Este resultado nos indica que efectivamente existe una tendencia a manifestar mayor ansiedad cuando se presenta un nivel relativamente bajo de asertividad; además, estas características son más distintivas del sexo femenino que del masculino, principalmente en el grupo chicano.

\section{CONCLUSIONES}

A partir de los resultados antes expuestos consideramos que, aunque no se puede afirmar de manera categórica, es posible plantear la relación propuesta entre asertividad y ansiedad. Pero más importante aún es el hecho de que la población chicana manifiesta con mayor dominancia dicha relación. La explicación más plausible a esta circunstancia es la exigencia de dos diferentes patrones culturales a un mismo sujeto.

Esta diferencia de patrones culturales puede ser o no real, empíricamente hablando, pero la información recabada por nosotros seffala que los sujetos están percibiendo situaciones que les ocasionan elevados niveles de ansiedad.

No hay que olvidar que la población con la que se trabajó es de nivel universitario, y que también puede suceder que esas situaciones que 
apuntamos arriba se complementen con la presión normal que existe en el transcurso de la formación profesional, aspecto que ha sido sefalado por otros autores (Sharma, Dang y Spielberger, 1986). En este punto se hace necesario continuar esta investigación con sujetos de diferentes niveles educativos.

Cualquier investigación que se proponga a futuro, debiera enfocarse, en primer lugar, y con base en la utilidad que se le encuentren a los datos aquí presentados, a explicar y encontrar los posibles factores que originan ese comportamiento característico en el grupo chicano, y mayormente dentro del sexo femenino.

Hasta aquí, podemos proponer que las poblaciones fronterizas identificadas presentan diferencias notables en relación con los grupos de poblaciones de las regiones céntricas del país, lo cual se refuerza con los resultados expuestos, que otorgan como característica particular al grupo de sujetos chicanos, un elevado nivel de ansiedad y un notable decremento en asertividad.

Consideramos que este trabajo, si bien contesta algunas interrogantes, plantea una línea de trabajo que debe pretender identificar las características psicologicas de los habitantes de zonas fronterizas, en especial del grupo chicano y de los emigrados en general, ya que hasta el momento consideramos que muchas de las caracterizaciones que se manejan - tanto por autoridades como por algunos "estudiosos" de escritorio- merecen ser contrastadas en forma directa, para posteriormente elaborar políticas que vayan de acuerdo al carácter nacional y local del habitante de una de las fronteras más largas de nuestro continente, y que - para bien o para mal- une dos países con niveles de desarrollo económico, político, social, cultural e histórico cualitativamente distintos.

\section{BIBLIOGRAFÍA}

BANDURA, A. 1975. Modificación de conducta: análisis de la agresión y la delincuencia. Ed. Trillas, México.

BARTOLOMÉ, Carrobles, Costa, Del Ser. 1976. Práctica de la terapia de la conducta. Ed. Pablo del Río, Barcelona.

BECK, A. 1976. Cognitive therapy and the emotional disorders. Ed. International University Press, San Francisco.

COLEMAN, J. C.; Butcher, J. N. y Carson, R. 1988. Psicología de la anormalidad y vida moderna. Ed. Trillas, México.

DIAZ-GUERRERO, R. 1982. Psicologia del mexicano. Ed. Trillas, México, cuarta edición. 
ELLIS, A. 1957. How to live with a neurotic. Ed. Crown, Nueva York. KERLINGER, F. N. 1975. Investigación del comportamiento. Técnicas y metodología. segunda edición. Ed. Interamericana, México.

LAZARUS, A. 1977. "Toward an egoless state of being". En: A. Ellis y H. Grieger. Handbook of RET. Ed. Springer Publishing Co., Nueva York.

McKAY, M.; Davis, M. y Fanning, P. 1985. Técnicas cognitivas para el tratamiento del estrés. Ed. Roca, México.

PADUA, J. 1979. Técnicas de investigación asociadas a las ciencias sociales. Ed. Fondo de Cultura Económica, México.

PAL-HEGEDUS, C. y Jensen, H. 1982. "Propiedades psicométricas y estructura factorial de la Escala de Asertividad de Rathus en una muestra de universitarios". Revista de la Asociación Latinoamericana de Psicologia Social, 2, no. 2.

PAZ, O. 1959. El laberinto de la soledad. Ed. Fondo de Cultura Económica, México.

RAMIREZ, S. 1977. El mexicano. Psicologia de sus motivaciones. Ed. Grijalbo, 4a. ed. México.

RATHUS, S.A. 1973. "Assesing Assertive Behavior". Behavior Therapy. 4. SARASON G., I. 1975. Psicologla anormal. Ed. Trillas, México.

SHARMA, S.; Dang, R. y Spielberger, Ch. 1986. "Effects of trait anxiety and intelligence on academic performance in different school courses". En: Ch. Spielberger y R. Díaz-Guerrero: Transcultural studies in anxiety. Ed. John Wiley, Boston.

SPIELBERGER, Ch. 1987. STA. State Trait Anxiety Inventory. Ed. Consulting Psychologists Press, Palo Alto.

SPIELBERGER, Ch. y R. Díaz-Guerrero. 1975. IDARE. Inventario de Ansiedad Rasgo Estado. Ed. El Manual Moderno, México.

- 1986. Transcultural studies in anxiety. Ed. John Wiley, Boston.

VILLANUEVA, T. 1985. Chicanos (selección). Ed. Fondo de Cultura Económica/sEP, colección Lecturas Mexicanas, México.

WAYNE, H.; R. Díaz-Guerrero; J. Swartz. 1975. Desarrollo de la personalidad en dos culturas: México y Estados Unidos. Ed. Trillas, México. 